\title{
Idade materna e mortalidade infantil: efeitos nulos, biológicos ou socioeconômicos?
}

\author{
Luciana Conceição de Lima*
}

\begin{abstract}
Há controvérsias se os extremos da idade reprodutiva feminina agregam o maior conjunto de fatores associados à mortalidade infantil, e se para filhos de mães abaixo dos 20 anos e a partir dos 35 anos de idade há evidências de maiores chances de ocorrência do óbito infantil. Para as mães adolescentes, há um importante debate se os resultados obstétricos adversos alcançados devemse à imaturidade biológica, às condições socioeconômicas desfavoráveis, ou se há nulidade do efeito da jovem idade sobre as chances de ocorrência da mortalidade infantil. Para mães em idades avançadas, discute-se se o aumento na idade materna, a despeito dos avanços no campo da medicina, está associado ao crescimento da vulnerabilidade ao óbito infantil, se as melhores condições socioeconômicas atenuam o efeito das comorbidades maternas comuns à idade de 35 anos ou mais, ou ainda se a maternidade tardia não exerce qualquer efeito sobre a ocorrência da mortalidade infantil. Com base em evidências contrastantes acerca das chances de ocorrência da mortalidade infantil por idade materna, este trabalho objetiva apresentar e discutir os principais argumentos evocados pela literatura. No caso das maternidades precoce e tardia, há evidências que corroboram ambas as hipóteses da plausibilidade biológica e socioeconômica e da nulidade do efeito da idade materna. A variedade de metodologias empregadas para o estudo da associação entre idade da mãe ao ter o filho e a mortalidade infantil, além da não uniformidade na definição dos grupos etários entre os diversos estudos, dificulta uma identificação mais precisa acerca desta vulnerabilidade.
\end{abstract}

Palavras-chave: Mortalidade infantil. Idade materna. Efeitos biológicos. Efeitos socioeconômicos. Tendências de fecundidade.

\section{Introdução}

A idade da mãe ao ter o filho constitui importante fator relacionado ao óbito infantil, sobretudo quando há precocidade ou postergação da maternidade ao longo do período reprodutivo feminino. Existem evidências de bipolarização das chances de ocorrência do óbito para filhos de mães muito jovens (menos de 20 anos) e de 35 anos e mais, em função de uma série de fatores comportamentais, socioeconômicos e biológicos (GUIMARÃES; VELÁSQUESMELÉNDEZ, 2002; BACAK et al., 2005; O'LEARY et al., 2007). Verifica-se, na literatura, uma importante discussão quanto ao fato de os resultados obstétricos adversos na população de mães adolescentes deverem-

\footnotetext{
* Socióloga, mestre e doutoranda em Demografia pelo Centro de Desenvolvimento e Planejamento Regional - Cedeplar, da Universidade Federal de Minas Gerais. Bolsista do CNPq - Brasil.
} 
se a uma possível imaturidade biológica, às condições socioeconômicas desfavoráveis, ou se é nulo o efeito da jovem idade materna sobre a vulnerabilidade ao óbito infantil. Também se discute se a maternidade nas idades avançadas está associada ao óbito de crianças abaixo de um ano de idade, se as melhores condições socioeconômicas que, em geral, estas mães experimentam atenuam o impacto das limitações biológicas naturais à gravidez após os 35 anos, e também se existe nulidade do efeito da idade materna avançada sobre a ocorrência de resultados obstétricos adversos (CALLAWAY; LUST; MCINTYRE, 2005; OLESZCZUK; KEITH; OLESZCZUK, 2005; LUKE; BROWN, 2007).

Com base em evidências contrastantes acerca dos efeitos da idade da mãe ao ter o filho, o objetivo deste trabalho é o de apresentar e discutir as principais tendências da fecundidade por idade da mãe e algumas das construções socioculturais e biomédicas das maternidades precoce e tardia, bem como algumas das correntes teóricas que fundamentam de maneira distinta as evidências de vulnerabilidade ao óbito infantil por idade materna. Por se tratar de uma revisão narrativa, este texto traz uma análise ampla e crítica da literatura sobre o tema abordado. Desse modo, não foram empregados métodos explícitos e sistemáticos para identificar e selecionar os estudos ora apresentados nesta revisão. As possíveis limitações/potencialidades advindas com a revisão de literatura empregada no presente trabalho também serão discutidas.

\section{A maternidade nas idades jovens}

Estima-se que, em todo o mundo, cerca de 14 milhões de adolescentes dão à luz anualmente e $90 \%$ desse total vivem em países em desenvolvimento (WORLD HEALTH ORGANIZATION, 2004). Na América Latina e no Caribe, apesar da tendência de queda na taxa de fecundidade total (TFT), quando se observa o comportamento reprodutivo por grupos etários, verifica-se que, nas últimas décadas, para o segmento de mulheres menores de 20 anos de idade, a fecundidade aumentou, especialmente entre aquelas com menos de 18 anos, embora o uso de métodos contraceptivos para esse grupo tenha se elevado (CENTRO LATINOAMERICANO Y CARIBEÑO DE DEMOGRAFÍA, 2005).

No Brasil, seguindo a tendência observada em boa parte da América Latina e do Caribe, a fecundidade iniciou um acelerado processo de declínio em meados da década de 1960, passando de uma TFT de 6,3 para 2,1 filhos por mulher, entre 1960 e 2004 (BERQUÓ; CAVENAGHI, 2006). Dados recentes da Pesquisa Nacional de Demografia e Saúde - PNDS 2006 indicaram uma TFT de 1,8 filho por mulher, nos 36 meses anteriores à data da pesquisa, para todo 0 Brasil (CENTRO BRASILEIRO DE ANÁLISE E PLANEJAMENTO, 2008). Verificou-se, também, aumento de $25 \%$ da taxa específica de fecundidade para mulheres de 15 a 19 anos, entre 1991 e 2000, ao passo que para os demais grupos etários femininos as taxas apresentam ritmo de queda consistente, o que tem se convertido no rejuvenescimento do padrão etário da fecundidade no Brasil (LEITE; RODRIGUES; FONSECA, 2004; BERQUÓ; CAVENAGHI, 2005; DIAS; AQUINO, 2006)

Alguns estudos apontam para uma tendência de declínio, a partir de 2000, da fecundidade entre mulheres de 15 a 19 anos, sobretudo nas regiões mais desenvolvidas do país, mas ainda persistem importantes diferenças entre grupos com características sociodemográficas distintas (BARBOSA, 2008; YAZAKI, 2008). Segundo informações do relatório da PNDS 2006, entre outras dimensões analisadas, o percentual de muIheres de 15 a 19 anos grávidas pela primeira vez, na data da entrevista, alcançou maiores valores para as jovens do meio urbano $(5,6 \%)$, em relação àquelas pertencentes ao meio rural $(2,4 \%)$, e para as adolescentes negras $(7,5 \%)$ quando comparadas às brancas $(4,8 \%)$ (CENTRO BRASILEIRO DE ANÁLISE E PLANEJAMENTO, 2008).

Todavia, a gravidez na adolescência tem se destacado como um potencial problema a ser resolvido. Além de merecer importância pelo fato de o contingente de adolescentes na população brasileira ainda ser expressivo, mesmo em meio ao processo de envelhecimento populacional, as discussões 
se dirigem para uma definição de qual seria a idade adequada à maternidade (se é que existe), bem como o melhor momento para se ter filhos (HEILBORN, 1998; HEILBORN et al., 2002).

No Brasil, no contexto da década de 1960, em que se notam importantes alterações de comportamento e de valores nas relações de gênero, houve também mudanças na concepção social das idades, redefinindo novas expectativas com relação à população feminina adolescente (HEILBORN, 1998). As oportunidades educacionais, profissionais e de vivência da sexualidade desvinculada do papel reprodutivo, que em princípio descortinaram-se para a população jovem, fundamentaram uma nova concepção da idade ideal à maternidade (HEILBORN et al., 2002). Porém, considerar a gravidez na adolescência um desperdício de tais oportunidades e um golpe na emancipação feminina pode significar que as oportunidades de acesso à educação, à qualificação profissional e ao exercício dos direitos reprodutivos alcançam indistintamente todos os grupos sociais (HEILBORN et al., 2002; PANTELIDES, 2004). Isto, entretanto, não pode ser aplicado ao Brasil, uma vez que parcela considerável de sua população jovem encontra-se alijada não apenas de informação e uso de métodos contraceptivos, mas também de oportunidades de escolha pelos estudos e pela carreira.

Com relação à abordagem da gravidez na adolescência como um problema social no século XX, deve-se ressaltar, inicialmente, que boa parte das sociedades ocidentais experimentou um aumento na incidência do intercurso sexual e de gravidezes entre adolescentes, em especial após a Segunda Guerra mundial (WORLD HEALTH ORGANIZATION, 2004). Em meados da década de 1970, nos países desenvolvidos, sobretudo nos Estados Unidos, entrou em cena um discurso biomédico que apontava as consequências deletérias do número crescente de gravidezes na adolescência sobre a saúde e o bem-estar da jovem mãe e do recém-nascido (PANTELIDES, 2004; WORLD HEALTH ORGANIZATION, 2004). No Brasil, assim como em boa parte dos países em desenvolvimento, igualmente nos anos 1970, o discurso biomédico de danos à saúde materno-infantil em virtude da gravidez na adolescência cedeu espaço para aquele que enfatizava a imaturidade psicológica das adolescentes para a maternidade (HEILBORN, 2002; PANTELIDES, 2004). Essas construções perpassaram a década de 1980 e se consolidaram na de 1990, arrolando uma série de argumentos cuja tônica era a de perpetuação de condições de vida miseráveis, decorrentes em grande medida da gravidez na adolescência (HEILBORN, 2002).

Em linhas gerais, a questão da maternidade nas jovens idades como um problema encontrou apoio ora na alegação da imaturidade biológica das mães adolescentes, ora na sustentação de que as condições socioeconômicas desfavoráveis conseguem, mais do que a idade por si mesma, explicar a ocorrência de tais resultados adversos. Entre os países desenvolvidos, os Estados Unidos, da década de 1970 até aproximadamente os anos 1990, destacavam-se por apresentar elevadas taxas de fecundidade no grupo de menores de 18 anos, tanto entre as jovens negras quanto entre as brancas e de status socioeconômico mais elevado do que as primeiras (SINGH; DARROCH, 2000). Havia uma associação entre o comportamento reprodutivo das adolescentes e as expressivas taxas de mortalidade infantil, sobretudo dos filhos das adolescentes negras, que experimentavam taxas mais elevadas do que os filhos das adolescentes brancas (GERONIMUS, 1987 e 2004). Esse relacionamento era interpretado do ponto de vista estritamente biológico, no qual a fecundidade precoce era tida como algo inerentemente deletério à sobrevivência dos filhos das adolescentes (GERONIMUS, 1987 e 2003).

Ainda não há consenso sobre em que medida a idade materna é capaz de explicar resultados obstétricos adversos. Um argumento relacionado à imaturidade biológica das jovens mães afirma que o desenvolvimento ainda em curso do organismo adolescente compete com o desenvolvimento fetal, conduzindo ao baixo peso ao nascer e à prematuridade, o que causa prejuízos à saúde do futuro recém-nascido (SCHOLL; 
HEDIGER; ANCES, 1990; CUNNINGTON, 2001; KING, 2003). Mesmo nos casos em que a gestante menor de 18 anos apresenta ganho de peso adequado e consegue acumular reservas de gordura suficientes, os recém-nascidos dessas jovens, ainda em fase de crescimento, costumam pesar menos do que os de gestantes que não apresentam desaceleração no crescimento intrauterino (SCHOLL et al., 1994; LUTHER et al., 2007).

Outro argumento a favor da hipótese da imaturidade biológica refere-se ao fato de que a jovem idade ginecológica (concepção no período de dois anos após a menarca) pode implicar chances elevadas de ocorrência da mortalidade infantil, neonatal e pósneonatal para crianças gestadas durante essa fase, devido à imaturidade do desenvolvimento uterino das mães adolescentes (FRASER; BROCKERT; WARD, 1995; CHEN et al., 2008). Há ainda a hipótese de que meninas, em especial aquelas submetidas a altos níveis de estresse psicossocial, tendam a experimentar a menarca precocemente e a iniciar mais cedo a atividade sexual e a maternidade, apresentando, assim, chances elevadas de darem à luz recém-nascidos de baixo peso (COALL; CHISHOLM, 2003).

Alguns trabalhos indicam maior efeito da jovem idade sobre as chances de intercorrências obstétricas, mesmo quando se comparam grupos maternos com características semelhantes, ou para a existência de efeitos independentes da idade materna sobre resultados obstétricos adversos. Utilizando dados de hospitais e maternidades do município de São Luiz do Maranhão, Simões et al. (2003) encontraram evidências de que filhos de mães com idade inferior a 18 anos apresentavam maiores chances de nascerem prematuros, com baixo peso e de morrerem antes de completarem o primeiro ano de vida, em comparação com os filhos de mães de idades de 18 e 19 anos e com características socioeconômicas semelhantes às primeiras. Estudo de Silva et al. (2003), também empregando dados de registros hospitalares do município de São Luiz do Maranhão, mostrou que, mesmo após o ajuste por variáveis de confundimento, como renda familiar, status marital e parturição, as chances de ocorrência de nascidos vivos pré-termos para filhos de primíparas menores de 18 anos se mantiveram significativas.

A maternidade na adolescência também é apontada como um comportamento adaptativo às situações de extrema adversidade. Segundo Geronimus (1991, 1992, 1996 e 2003), nos Estados Unidos nas décadas de 1970 e 1980, e exclusivamente para as mães negras, que representam um grupo vulnerável do ponto de vista socioeconômi$\mathrm{co}$, a taxa de mortalidade infantil dos filhos das adolescentes apresentava-se menor do que a dos filhos de mães que estavam na segunda década de vida, um resultado consistente com a hipótese de que a maternidade precoce pode ser um elemento adaptativo ao processo, igualmente prematuro, de deterioração da saúde de mulheres negras.

Nos Estados Unidos, em reação ao discurso biomédico que defende a hipótese da imaturidade biológica de mães adolescentes, alguns estudos apontam que, ao se incorporarem nos modelos variáveis socioeconômicas, a associação entre idade materna e mortalidade infantil torna-se mais fraca, ou até mesmo desaparece (DAVANZO; BUTZ; HABICHT, 1983; GERONIMUS, 1987 e 2004). Fatores como baixa frequência às consultas de pré-natal, tabagismo, má nutrição, baixos níveis de renda e de escolaridade e ausência de uniões estáveis são comuns entre gestantes adolescentes (CHEN et al., 2007), o que pode explicar a grande ocorrência de resultados adversos para recém-nascidos dessas mães, tais como baixo peso, prematuridade e maiores chances de mortalidade neonatal e pós-neonatal (ALMEIDA et al., 2002; MOHSIN; BAUMAN; JALALUDIN, 2006; CHEN et al., 2007; HALDRE et al., 2007; REIME; SHÜCKING; WENZLAFF, 2008).

Utilizando dados para o município de Belo Horizonte, em 1993, César, MirandaRibeiro e Abreu (2000) obtiveram resultados que apontam a condição socioeconômica como o fator mais importante para explicar a ocorrência da mortalidade neonatal entre filhos de mães com idade inferior a 20 anos. Ao analisar o relacionamento entre mortalidade neonatal e gravidez na adolescência 
em uma área rural do Nepal, Sharma et al. (2008) verificaram que a inclusão de variáveis socioeconômicas no modelo, como escolaridade da mãe, atenuou de forma bastante significativa essa associação.

Há autores que advogam a hipótese de que a maternidade na adolescência não implica maior vulnerabilidade aos resultados obstétricos adversos do que a maternidade em outras fases do período reprodutivo feminino. Lawlor e Shaw (2002) defendem que mães adolescentes não apresentam desvantagens biológicas e socioeconômicas em relação àquelas de outros grupos etários. Além disso, os autores sugerem que a gama de resultados conflitantes existentes na literatura referente ao tema reflete, entre outros aspectos, a dificuldade dos estudiosos em separar efeitos da idade materna de efeitos decorrentes de variáveis de confusão. Apesar de terem encontrado maiores chances de nascimentos pré-termos para filhos de mães menores de 18 anos, Jolly et al. (2000) verificaram, segundo registros hospitalares de uma região no Reino Unido, que a proporção de recém-nascidos tanto pequenos quanto grandes para a idade gestacional foi a mesma para o grupo de mães adolescentes e o daquelas com 18 a 34 anos. No estudo de casos e controles de Jobim e Aerts (2008), para o município de Porto Alegre, a jovem idade materna não esteve associada a resultados obstétricos adversos, apesar de $24,1 \%$ dos óbitos terem sido registrados para filhos de mães com menos de 20 anos.

\section{A maternidade nas idades avançadas}

A idade materna avançada é estabelecida, tradicionalmente, como aquela igual ou superior a 35 anos (GUSMÃO, TAVARES; MOREIRA, 2003; KRISTENSEN et al., 2007), embora alguns autores apontem que a faixa etária de 40 anos e mais define o termo de forma mais adequada, tendo em vista os fatores associados relacionados mais fortemente à saúde da mãe e do recém-nascido nestas idades (CALLAWAY; LUST; MCINTYRE, 2005; CHAN; LAO, 2008). A experiência da maternidade em tais idades configura-se como uma ten- dência observável não apenas em países desenvolvidos, mas também naqueles em desenvolvimento, sendo sua prevalência mais elevada, sobretudo, no grupo de mulheres mais escolarizadas e de maior posse de recursos financeiros (YÁNES, 2007; CHAN; LAO, 2008).

Nos países desenvolvidos, aproximadamente entre o início do século XX e a década de 1970, o processo de declínio da fecundidade veio acompanhado da tendência de redução da idade média das mulheres ao terem seus filhos (ESHRE CAPRI WORKSHOP GROUP, 2005). A partir de então, essa tendência começou a se inverter e ganhos na idade média das mães passaram a ser observados em função, sobretudo, da postergação do nascimento do primeiro filho (ESHRE CAPRI WORKSHOP GROUP, 2005). Na Suécia, entre as primíparas, a idade média das mães passou de 24 para 28 anos, entre 1973 e 2003, e, no Canadá, de 28,8 para 29,6 anos, no período de 1995 a 2003 (MONTAN, 2007; BENZIES, 2008). Em 2003, a idade média das primíparas era de 28,3 anos na Holanda e de 24,9 anos nos Estados Unidos (BENZIES, 2008).

Na América Latina e no Caribe, por sua vez, no último quinquênio do século $X X$, a TFT não apenas decresceu, mas também houve um rejuvenescimento da fecundidade, com deslocamento do grupo modal, de 25 a 29 anos para o de 20 a 24 anos (FERRANDO, 2003). Seguindo a tendência dessa região, no Brasil, entre 1980 e 2000, os nascimentos vivos passaram a se concentrar no grupo etário de 20 a 24 anos, ao passo que o de 35 anos e mais diminuiu consideravelmente seu peso relativo nas taxas de fecundidade correntes, no período analisado (BERQUÓ; CAVENAGHI, 2005). A idade média das mães brasileiras passou de 25,6 anos, em 1991, para 24,8 anos, em 2000, e o grupo etário de 15 a 19 anos experimentou, no mesmo período, uma variação positiva de $25,4 \%$ na taxa de fecundidade, ao passo que, para todos os demais grupos etários, verificou-se variação negativa, em especial para os de 35 a 39 anos, 40 a 44 anos e 45 a 49 anos, com declínios, respectivamente, de $28 \%$, 47,8\% e $63,3 \%$ (OLIVEIRA, 2005; BASSI, 2008). 
No Brasil, assim como na América Latina e no Caribe, prevalecem padrões de entrada precoce ao casamento e de início do intercurso sexual, o que contribui para a concentração da fecundidade nos grupos etários maternos mais jovens (FERRANDO, 2003; SIMÃO, 2008). No que diz respeito ao total de mães pela primeira vez, na década de 1990, observou-se aumento da participação na faixa etária de 40 a 49 anos e, embora se saiba que esse ganho pouco tem contribuído para o envelhecimento do padrão etário da fecundidade, essa tendência revela o comportamento reprodutivo de um grupo que apresenta características socioeconômicas e comportamentais peculiares, em comparação àquelas em idades mais jovens (OLIVEIRA, 2005).

Alguns trabalhos apontam que mulheres que se tornam mães após os 35 anos, em geral, apresentam condições socioeconômicas favoráveis, recebem atendimentos pré-natal e obstétrico adequados e exercem maior planejamento econômico e emocional para o nascimento do primeiro filho (STEIN; SUSSER, 2000; SENESI et al., 2004; KRISTENSEN, 2007). A postergação do casamento e a constituição de novas uniões, os investimentos em educação e na carreira profissional, a ampliação do uso de métodos contraceptivos e problemas de infertilidade são fatores que contribuem para o adiamento da maternidade (TOUGH et al., 2002).

Para as mulheres, a passagem pelo período reprodutivo se insere em um contexto de expectativas sociais pelo desempenho simultâneo dos papéis ligados à maternidade e à carreira profissional, quando se inicia a vida adulta (TAIN, 2005). Segundo Guedes (2008), os tempos biológicos e sociais estão em permanente conflito e/ou negociação no universo feminino, sendo que o fato de as conquistas das mulheres no mercado de trabalho não virem acompanhadas por um processo de "desnaturalização" dos papéis tradicionalmente atribuídos às mesmas pode contribuir para que a maternidade seja deixada para mais tarde.

São arroladas outras estratégias para resolver o impasse entre a opção pela carreira e os filhos, como o abandono da carreira profissional em prol da maternida- de, a procura por empregos com horários de trabalho flexíveis para compatibilizar os afazeres domésticos com os profissionais e a opção pela profissão em detrimento da escolha de se ter filhos (DIAS JÚNIOR, 2007). Apesar de a escolha mais empregada ser aquela que de alguma forma sacrifica a carreira profissional, como a preferência por empregos com jornadas de trabalho reduzidas (TAIN, 2005), a postergação do nascimento do primeiro filho, até certo limite da idade, também é vista como uma forma de adquirir estabilidade financeira e emocional, que muitas consideram condições necessárias para se tornarem mães (OLIVEIRA; MARCONDES, 2004).

$O$ alcance de níveis educacionais elevados pode representar um estímulo para o adiamento do nascimento do primeiro filho, pois, quanto maior a escolaridade, maior a tendência de que a primeira relação sexual não aconteça precocemente, que a entrada no casamento seja postergada, que o uso de métodos contraceptivos seja maior e que se valorize a constituição de famílias menores (SIMÃO, 2005).

Resultados de um estudo empreendido em 13 países da América Latina e Caribe indicaram forte relação entre aumento da escolaridade feminina e início tardio tanto do intercurso sexual quanto da nupcialidade e da maternidade, com pouca variação entre os países analisados, entre os quais se inclui o Brasil (HEATON; FORSTE; OTTERSTROM, 2002).

Em muitas culturas, a entrada em união formal ou informal marca o início da constituição familiar e exerce importante influência sobre a fecundidade, uma vez que se supõe que mulheres unidas estão sujeitas a maior regularidade de relações sexuais, que as expõem, de maneira mais efetiva, ao risco de ter filhos (BAY; DEL POPOLO; FERRAN$D O, 2003)$. Ainda que os nascimentos não estejam, necessariamente, circunscritos à esfera do casamento, a nupcialidade exerce um importante papel sobre o número de filhos tidos, e aquelas que se casam tardiamente tendem, também, a adiarem o nascimento do primeiro filho (SIMÃO, 2005). Com relação à constituição de novas uniões, embora a fecundidade tenda a ser menor 
entre os recasados (MARCONDES, 2008), esse padrão de nupcialidade pode estar associado à postergação do encerramento da fase reprodutiva feminina.

No que tange à contracepção, mulheres que utilizam métodos contraceptivos modernos e de maneira mais sistemática tendem a adiar o nascimento do primeiro filho (SIMÃO, 2005). Informações do relatório da PNDS 2006 revelam que $80 \%$ das mulheres unidas no Brasil utilizavam algum método contraceptivo na data da pesquisa e apenas 3\% delas recorriam a métodos tradicionais (CENTRO BRASILEIRO DE ANÁLISE E PLANEJAMENTO, 2008).

Com relação à infertilidade, estima-se que nos países em desenvolvimento cerca de 186 milhões de casais são afetados por este problema, sendo que as principais causas são as infecções dos órgãos reprodutores provocadas por doenças como gonorreia e clamídia (WORLD HEALTH ORGANIZATION, 2003). A demanda por técnicas de tratamento da infertilidade tem aumentado nas últimas décadas, em decorrência, também, de mudanças comportamentais, como a postergação da maternidade para idades mais avançadas, quando o potencial reprodutivo de homens e especialmente das mulheres cai naturalmente (BRAZ; SCHRAMM, 2005). A idade é considerada o principal fator que limita o tratamento da infertilidade e, para as mulheres, a fertilidade declina após os 30 anos e de forma mais abrupta depois dos 40 anos (PASQUALOTTO; BORGES JÚNIOR; PASQUALOTTO, 2008).

$\mathrm{Na}$ América Latina, quase todos os países dispõem de legislação ou regulação formal para aplicação de técnicas de reprodução medicamente assistidas. Porém, em geral, esses tratamentos não estão disponíveis na rede pública, sendo acessados, basicamente, por uma clientela de poder aquisitivo elevado e em serviços de saúde especializados da rede particular (WORLD HEALTH ORGANIZATION, 2003).

Ainda no que se refere à fertilidade feminina, a perda de potencial reprodutivo se apresenta como aspecto inevitável com o avançar da idade (ESHRE CAPRI WORKSHOP GROUP, 2005) e nem sempre pode ser compensado com a utilização de técnicas de reprodução medicamente assistidas. Estudos indicam que esses métodos conseguem compensar, aproximadamente, menos de $30 \%$ da capacidade reprodutiva perdida por mulheres que postergam 0 nascimento do primeiro filho da idade de 35 para a de 40 anos (LERIDON, 2004; ESHRE CAPRI WORKSHOP GROUP, 2005). Mesmo entre as mulheres que não utilizam tais tecnologias, a taxa de sucesso da concepção (gravidezes terminadas em nascidos vivos por 100 mulheres de cada grupo etário) é substancialmente menor após os 40 anos (MAHESHWARI; HAMILTON; BHATTACHARYA, 2008).

No que se refere às morbidades maternas relacionadas à gravidez em idades avançadas, problemas como hipertensão e diabetes são alguns dos mais frequentes (JACOBSSON; LADFORS; MILSOM, 2004; LUKE; BROWN, 2007). Costa, Costa e Costa (2003) observaram, com base em dados de uma maternidade no Recife, que a idade materna igual ou superior a 40 anos representou fator associado à hipertensão induzida pela gravidez, independentemente da parturição e da presença de hipertensão arterial prévia e do diabetes. No que diz respeito ao relacionamento entre desordem hipertensiva e mortalidade infantil, os resultados de alguns trabalhos apontam maiores chances de ocorrência da mortalidade neonatal para filhos de mães com esse problema na gestação (ARAÚJO et al., 2005; DISSANAYAKE et al., 2007).

As perdas fetais também são indicadas na literatura como fatores associados à mortalidade infantil, mediadas pela idade materna avançada, embora não haja concordância estabelecida sobre quais mecanismos biológicos desencadeiam os óbitos fetais com maior frequência nas idades mais avançadas (HUANG et al., 2008). Apesar de ainda não serem bem conhecidas as implicações de históricos de perdas sobre os resultados obstétricos, no caso específico de abortos induzidos, uma das possíveis consequências desses eventos são os nascimentos pré-termos e de baixo peso, que representam um dos principais fatores de risco do óbito abaixo de um ano de vida 
(RAATIKAINEN; HEISKANEN; HEINONEN, 2006). Alguns trabalhos indicam também que recém-nascidos de primíparas de 35 anos ou mais são mais propensos a serem prematuros, pequenos para a idade gestacional, de baixo peso e com baixo índice de Apgar no 10 minuto (SENESI et al., 2004; CHAN; LAO, 2008).

Com relação à multiparidade, há evidências de que esta característica esteja associada, especialmente no caso das grandes multíparas (mulheres com cinco filhos nascidos vivos e mais), ao aumento das chances de ocorrência de abortos, desnutrição e anemia, gemelaridade, hemorragia anteparto e prematuridade do recém-nascido (RAYAMAJHI; THAPA; PAN$D E, 2006)$. A multiparidade está relacionada à idade materna avançada, sobretudo nos países em desenvolvimento, onde fatores culturais, como o rígido seguimento a certas doutrinas religiosas, e socioeconômicos, como as desigualdades de oportunidades educacionais, diminuem as chances de utilização de métodos contraceptivos para planejamento familiar, tornando as mulheres desse grupo mais vulneráveis a resultados adversos em suas respectivas gestações (RAYAMAJHI; THAPA; PANDE, 2006).

A postergação da maternidade também pode estar associada a intervalos intergenésicos curtos, implicando resultados obstétricos adversos para as gravidezes subsequentes (NABUKERA et al., 2008), já que nestes casos são grandes as chances de ocorrência de depleção materna, caracterizada pelo esgotamento nutricional devido às sucessivas gravidezes e aleitamentos (CONDE-AGUDELO; ROSAS-BERMÚDEZ; KAFURY-GOETA, 2006).

A idade materna avançada também se relaciona aos hábitos saudáveis durante a gestação (MACHADO; HILL, 2003), o que está ligado, em grande medida, à adesão às recomendações prescritas durante o pré-natal. Oleszczuk, Keith e Oleszczuk (2005) explicam que mães de faixas etárias mais avançadas costumam suspender com maior frequência o uso de álcool e de tabaco e seguir dietas balanceadas, o que auxilia no alcance de resultados obstétricos mais favoráveis. Além disso, o acompanhamento adequado do curso da gravidez, em geral apresentado por estas mães, contribui para a redução da prevalência de baixo peso ao nascer, minimizando a atuação de fatores associados (MINAGAWA et al., 2006).

Alguns autores defendem que a idade materna avançada não exerce influência sobre os resultados obstétricos. Ao contrário, essas características positivas de conduta com a saúde pré-natal e as maiores chances de condições socioeconômicas e psicológicas favoráveis habilitam essas mães a experimentaram gravidezes com resultados obstétricos favoráveis. Para Marasinghe, Karunananda e Amarasinghe (2007), à parte de fatores como a senescência ovariana e a ocorrência de desordens genéticas, como anomalias cromossômicas, a idade avançada por si só não deve ser considerada um indicador de resultados obstétricos adversos, sendo equívoco afirmar que os recém-nascidos dessas mulheres apresentam maiores chances de experimentarem eventos como o óbito neonatal, o baixo peso ao nascer e a prematuridade. Callaway, Lust e Mcintyre (2005) estudaram os resultados de gravidezes de uma coorte de mulheres australianas de 45 anos e mais e verificaram que as puérperas que conceberam naturalmente, sem recorrer às técnicas médicas de reprodução, apresentaram resultados obstétricos favoráveis, possivelmente em decorrência de um prévio bom status de saúde.

\section{Limitações}

Por se tratar de um artigo de revisão narrativa, o objetivo precípuo deste estudo foi o de descrever e discutir os efeitos da idade materna sobre a mortalidade infantil, com base na consulta de trabalhos sobre o tema, sem orientar a busca destas fontes em métodos predeterminados ou específicos de seleção (CORDEIRO et al., 2007; ROTHER, 2007). Em linhas gerais, uma revisão narrativa é constituída pela análise crítica e pessoal do autor de literatura publicada em livros, revistas e material eletrônico. Ao contrário das revisões sistemáticas que utilizam métodos explícitos e sistemáticos para identificar, selecionar e avaliar criticamente 
os estudos que serão incluídos, a seleção dos artigos na revisão narrativa é arbitrária e sua elaboração não está pautada em protocolos rígidos (COLLINS; FAUSE, 2005; CORDEIRO et al., 2007). Tendo em vista que a busca das fontes é menos abrangente do que nas revisões sistemáticas e diante da ausência de métodos de seleção dos artigos incluídos, as revisões narrativas apresentam problemas, como vieses de seleção e ausência de metodologia que permita a reprodução dos dados e o acesso aos resultados quantitativos (COLLINS; FAUSE, 2005; ROTHER, 2007).

Assim, por se tratar de uma revisão narrativa, o presente trabalho não está isento de conter vieses de seleção dos artigos que o compõem, sendo que a não apresentação da metodologia e dos resultados quantitativos de cada artigo incluído não favoreceu o estabelecimento de uma discussão mais detalhada acerca dos diferenciais de mortalidade infantil por idade materna, o que poderia ser bastante elucidativo para a identificação das idades jovens e avançadas em que a vulnerabilidade ao óbito infantil torna-se mais premente. Por exemplo, no caso específico das mães adolescentes, os resultados obstétricos adversos exibidos por essa população, mesmo após o ajuste por importantes fatores de confundimento, podem ser o reflexo de atitudes particulares de um determinado grupo social com relação à maternidade nas idades jovens (LAWLOR; SHAW, 2002), o que torna a análise ainda mais complexa se são estabelecidas comparações entre trabalhos de diversas localidades e que utilizaram diferentes estratégias para a condução dos resultados.

\section{Conclusão}

Neste trabalho de revisão narrativa acerca dos diferenciais de mortalidade infantil por idade materna, foram apresentadas e discutidas, de maneira crítica, algumas hipóteses relacionadas a esses diferenciais. Conforme exposto, é possível identificar, na literatura, evidências a favor de explicações de naturezas biológica e socioeconômica e até mesmo a defesa da neutralidade da idade materna para explicar as chances de ocorrência da mortalidade infantil para filhos de mães muito jovens e, também, nas idades avançadas.

Do ponto de vista metodológico, devese considerar a variedade de métodos, ferramentas de análise e a não uniformidade na definição dos grupos etários entre diversos trabalhos, o que torna ainda mais difícil estabelecer comparações e chegar a uma melhor definição sobre a idade na qual os resultados obstétricos adversos tornam-se mais elevados. Por se tratar de uma revisão narrativa, o presente estudo não incluiu os resultados e a metodologia empregada por todos os trabalhos relacionados, que, ademais, não foram selecionados com base em métodos específicos e sistemáticos. Assim, sugere-se, para trabalhos futuros, a elaboração de revisões sistemáticas para minimizar o efeito de possíveis vieses no trabalho de identificação, seleção e análise crítica dos estudos relativos aos diferenciais de mortalidade infantil por idade materna. O emprego de métodos estatísticos (meta-análise) também pode ser uma boa estratégia em trabalhos futuros para análise e síntese mais detalhada dos resultados de diversos estudos, o que pode conduzir a importantes conclusões sobre as idades maternas em que as chances de ocorrência do óbito infantil se tornam mais elevadas.

No que diz respeito às mães adolescentes, a construção social da maternidade antes dos 20 anos de idade e sua variação de acordo com normas e sanções culturais praticadas por um determinado grupo social também figuram como elementos importantes para compreensão do fenômeno, que devem ser contemplados nas análises. Em boa parte dos estudos, utiliza-se o grupo de mães de 15 a 19 anos para caracterizar a maternidade na adolescência, sobretudo pela dificuldade de se captar um número expressivo de mães com 14 anos ou menos. Todavia, verifica-se que há importantes diferenças em se considerar o limite inferior da faixa etária materna menor do que o usualmente utilizado (PHIPPS; SOWERS, 2002), o que chama a atenção para o fato de que as chances de ocorrência da mortalidade infantil associadas à jovem idade materna podem ser subestimadas quando 
são empreendidas análises de faixas etárias muito agregadas. Assim, sugere-se que, em trabalhos futuros, sejam analisados grupos etários menos agregados, com o objetivo de verificar se os riscos de mortalidade infantil se comportam de maneira distinta, para faixas etárias menos heterogêneas. Contudo, é necessário ressaltar que esta tentativa de desagregação requer formas de análise apropriadas para que se possa lidar com pequenos números.

No caso da maternidade nas idades avançadas, tradicionalmente, são incluídas neste grupo as mães de 35 anos e mais (YÁNEZ, 2007; REDE INTERAGENCIAL DE INFORMAÇÕES PARA A SAÚDE, 2008a). Porém, sobretudo nos países desenvolvidos, cuja proporção de mulheres que se tornam mães nessa faixa etária é bem elevada, há distinção das chances de ocorrência de resultados obstétricos adversos entre mulheres de 40 a 44 anos e de 45 anos e mais (DONOSO; BECKER; VILLARROEL, 2002; JACOBSSON; LADFORS; MILSON, 2004; LUKE; BROWN, 2007), o que também chama a atenção para a análise de faixas etárias mais desagregadas para identificar aquelas idades cujas chances de ocorrência do óbito infantil, possivelmente, se tornam mais elevadas.

Ainda com relação à maternidade após os 35 anos de idade, cabe ressaltar a impor-

\section{Referências}

ALMEIDA, M. F. et al. Mortalidade neonatal no município de São Paulo: influência de peso ao nascer e de fatores sóciodemográficos e assistenciais. Revista Brasileira de Epidemiologia, São Paulo, v. 5, n. 1, p. 62-76, abr. 2002.

ARAÚJO, B. F. Estudo da mortalidade de recém-nascidos internados na UTI neonatal do Hospital Geral de Caxias do Sul, Rio Grande do Sul. Revista Brasileira de Saúde Materno Infantil, Recife, v. 5, n. 4, p. 463469, out./dez., 2005.

BACAK, S.J. et al. Risk factors for neonatal mortality among extremely-low-birth-weight infants. American Journal of Obstetrics tância em se explorar o efeito das possíveis melhores condições socioeconômicas, emocionais e comportamentais, em geral, atribuídas às mães em idades avançadas, para minimizar a atuação de comorbidades típicas da idade materna avançada, como diabetes e hipertensão arterial, que podem estar relacionadas ao óbito infantil. A maior utilização de métodos de reprodução medicamente assistida, advinda com a tendência de postergação da maternidade, também instiga as análises da ocorrência de óbito infantil para filhos de mães que não conceberam naturalmente.

Por fim, este artigo contribui para um debate que ainda suscita muitas indagações acerca da associação entre a idade da mãe ao ter o filho e o óbito entre menores de um ano de idade. Sobretudo no caso do Brasil, cujas taxas de mortalidade infantil ainda se encontram em patamares elevados, se comparadas àquelas já alcançadas pelos países desenvolvidos e por alguns países da América Latina, é de grande importância identificar e avaliar níveis e tendências do óbito neonatal (precoce e tardio) e pós-neonatal. Ainda não há concordância estabelecida na literatura quanto à natureza dos efeitos da idade materna sobre as chances de ocorrência do óbito infantil, o que abre espaço para futuras discussões.

\& Gynecology, Saint Louis, v. 192, n. 3, p. 862-867, Mar. 2005.

BARBOSA, G. P. et al. Parto cesáreo: quem o deseja? Em quais circunstâncias? Cadernos de Saúde Pública, Rio de Janeiro, v. 19, n. 6, p. 1.611-1.620, dez. 2003.

BAY, G.; DEL POPOLO, F.; FERRANDO, D. Determinantes próximos de la fecundidad. Una aplicación a países latinoamericanos. In: LA FECUNDIDAD EN AMÉRICA LATINA: ¿TRANSICIÓN O REVOLUCIÓN?, 43., 2003, Santiago de Chile. Anais... Santiago de Chile: Cepal, 2003.

BENZIES, K. M. Advanced maternal age: are decisions about the timing of child-bearing 
a failure to understand the risks? Canadian Medical Association Journal, Ottawa, v. 178, n. 2, p. 183-184, Jan. 2008.

BERQUÓ, E.; CAVENAGHI, S. Breve nota sobre a redução no número médio de filhos por mulher no Brasil. Novos Estudos Cebrap. São Paulo, v. 74, mar. 2006.

. Increasing adolescent and youth fertility in brazil: a new trend or a one-time event? In: THE ANNUAL MEETING OF THE POPULATION ASSOCIATION OF AMERICA, 2005, Philadelphia. Anais... Pennsylvania: Population Association of America, 2005.

BRAZ, M.; SCHRAMM, F. R. O ninho vazio: a desigualdade no acesso à procriação no Brasil e a bioética. Revista Brasileira de Bioética, Brasília, v.1, n. 2, 2005.

CALLAWAY, L. K.; LUST, K.; MCINTYRE, H. $D$. Pregnancy outcomes in women of very advanced maternal age. Australian and New Zealand Journal of Obstetrics and Gynaecology, Melbourn, v.45, n.1, p. 12-16, Feb. 2005.

CENTRO BRASILEIRO DE ANÁLISE E PLANEJAMENTO - CEBRAP. Pesquisa Nacional de Demografia e Saúde da Mulher: PNDS 2006. Brasília: Ministério da Saúde, 2008. Relatório.

CENTRO LATINOAMERICANO Y CARIBEÑO DE DEMOGRAFÍA. Fecundidad: una región em la que nacen menos niños. 2005. (Comportamiento Reproductivo, 2). Disponível em: <http://www.eclac.cl/celade/ noticias/noticias/2/23462/PyDCR_2.pdf $>$. Acesso em: 15 nov. 2008.

CÉSAR, C. C.; MIRANDA-RIBEIRO, P.; ABREU, D. M. X. Efeito-idade ou efeitopobreza? Mães adolescentes e mortalidade neonatal em Belo Horizonte. Revista Brasileira de Estudos de População, Campinas, v.17, n.1/2, p.177-196, jan./dez. 2000.

CHAN, B. C.; LAO, T.T. Effect of parity and advanced maternal age on obstetric outcome. International Journal of Gynecology \& Obstetrics, Ireland, v. 102, n. 3, p. 237-241, Sep. 2008.

CHEN, X. K. et al. Increased risks of neonatal and postneonatal mortality associated with teenage pregnancy had different explanations. Journal of Clinical Epidemiology, New York, v. 61, n. 7, p. 688694, Jul. 2008.

CHEN, X.K. et al. Teenage pregnancy and adverse birth outcomes: a large population based retrospective cohort study. International Journal of Epidemiology, Oxford, v. 36, n. 2, p. 368-373, Apr. 2007.

COALL, D. A.; CHISHOLM, J. S. Evolutionary perspectives on pregnancy: maternal age at menarche and infant birth weight. Social Science \& Medicine, v.57, 2003.

COLLINS, J. A.; FAUSER, B. C. Balancing the strengths of systematic and narrative reviews. Human Reproduction Update, v.11, n.2, p. 103-4, Mar.-Apr. 2005.

CONDE-AGUDELO, A.; ROSAS-BERMÚDEZ, A.; KAFURY-GOETA, A. C. Birth spacing and risk of adverse perinatal outcomes: a metaanalysis. Journal of the American Medical Association, Chicago, v. 295, n. 15, p. 1.8091.823, Apr. 2006.

CORDEIRO, A. M. et al. Revisão sistemática: uma revisão narrativa. Revista do Colégio Brasileiro de Cirurgióes, Rio de Janeiro, v. 34, n. 6, dec. 2007.

COSTA, H. L. F. F.; COSTA, C. F. F.; COSTA, L. O. B. F. Idade materna como fator de risco para a hipertensão arterial induzida pela gravidez: análise multivariada. Revista Brasileira de Ginecologia e Obstetrícia, Rio de Janeiro, v. 25, n. 9, p. 631-635, set. 2003.

CUNNINGTON, A. J. What's so bad about teenage pregnancy? Journal of Family Planning and Reproductive Health Care, England, v. 27, n. 1, p. 36-41, Jan. 2001.

DAVANZO, J.; BUTZ, W. P.; HABICHT, J. P. How biological and behavioral influences on mortality in Malaysia vary during the first year of life. Population Studies, London, v. 37, n. 3, p. 381-402, Nov. 1983.

DIAS JÚNIOR, C. S. Comportamento reprodutivo: uma análise a partir do grupo ocupacional das mulheres. Tese (Doutorado em Demografia) - Centro de Desenvolvimento e Planejamento Regional, 
Universidade Federal de Minas Gerais, Belo Horizonte, 2007.

DIAS, A. B.; AQUINO, E. M. L. Maternidade e paternidade na adolescência: algumas constatações em três cidades do Brasil. Cadernos de Saúde Pública, Rio de Janeiro, v. 22, n. 7, p. 1.447-1.458, jul. 2006.

DISSANAYAKE, V.H. et al. Morbidity and mortality associated with pre-eclampsia at two tertiary care hospitals in Sri Lanka. The Journal of Obstetrics and Gynaecology Research, Tokyo, v. 33, n.1, p. 56-62, Feb. 2007.

DONOSO, S. E.; BECKER V. J.; VILLARROEL, D. P. L. Evolucion de la natalidad y del riesgo reproductivo en mujeres de 40 o mas años en la decada de los 90. Revista Chilena de Obstetricia y Ginecología, Santiago, v. 67, n. 2, p. 139-142, 2002.

ESHRE CAPRI WORKSHOP GROUP. Fertility and ageing. Human Reproduction Update, Oxford, v. 11, n. 3, p. 261-276, May./Jun. 2005.

FERRANDO, D. La fecundidad por edades en América Latina y sus perspectivas futuras. In: SEMINARIO LA FECUNDIDAD EN AMÉRICA LATINA: TRANSICIÓN O REVOLUCIÓN? 36. Anais... Santiago de Chile: Cepal, 2003.

FRASER, A. M.; BROCKERT, J. E.; WARD, R. H. Association of young maternal age with adverse reproductive outcomes. New England Journal of Medicine, Waltham, v. 332, n.17, p. 1.113-1.120, Apr.1995

GERONIMUS, A. T. Black/white differences in the relationship of maternal age to birthweight: A population-based test of the weathering hypothesis. Social Science \& Medicine, Oxford, v. 42, n. 4, p. 589-597, Feb. 1996.

Damned if you do: culture, identity, privilege, and teenage childbearing in the United States. Social Science and Medicine, Oxford, v. 57 , n. 5 , p. $881-893$, Sep. 2003.

On teenage childbearing and neonatal mortality in the United States. Population and Development Review, New York, v. 13, n. 2, p. 245-279, Jun. 1987.
Teenage childbearing and social and reproductive disadvantage: the evolution of complex questions and the demise of simple answers. Family Relations, United Kingdom, v. 40, n. 4, p. 463-471, Oct. 1991.

. Teenage childbearing and social disadvantage: unprotected discourse. Family Relations, United Kingdom, v. 41, n. 2, p.244-248, Apr. 1992.

Teenage childbearing as cultural prism. British Medical Bulletin, Edinburgh, v. 69, n. 1, p.155-166. 2004.

GUEDES, M. C. Mulheres de alta escolaridade: repensando a relação entre maternidade e mundo do trabalho. In: ENCONTRO NACIONAL DE ESTUDOS POPULACIONAIS, 16. Anais... Caxambu: Abep, 2008.

GUIMARÃES, E. A. de A.; VELÁSQUEZMELÉNDEZ, G. Low birth weight determinants from the Born Alive National Surveillance System in Itaúna, Minas Gerais. Revista Brasileira de Saúde Materno Infantil, Recife, v. 2, n. 3, p. 283-290, set./dez. 2002.

GUSMÃO, F. A. F.; TAVARES, E. J. M.; MOREIRA, L. M. de A. Idade materna e síndrome de Down no Nordeste do Brasil. Cadernos de Saúde Pública, Rio de Janeiro, v. 19, n. 4, p. 973-978, jul./ago. 2003.

HALDRE, K. et al. A poor pregnancy outcome related to young maternal age? A study of teenagers in Estonia during the period of major socio-economic changes (from 1992 to 2002). European Journal of Obstetrics, Gynecology and Reproductive Biology, Amsterdam, v. 131, n. 1, p. 45-51, Mar. 2007.

HEATON, T. B.; FORSTE, R.; OTTERSTROM, S. M. Family transitions in Latin America: first intercourse, first union and first birth. International Journal of Population Geography, Chichester, v.8, n. 1, p.1-15, Jan./Feb. 2002.

HEILBORN, M. L. et al. Aproximações socioantropológicas sobre a gravidez na adolescência. Horizontes Antropológicos, Porto Alegre, v. 8, n. 17, p. 13-45, jun. 2002.

Gravidez na adolescência: considerações preliminares sobre as dimensões 
culturais de um problema social. In: SEMINÁRIO GRAVIDEZNA ADOLESCÊNCIA. Anais... Rio de Janeiro: Cepia/lpea, 1998, p. 23-32.

HUANG, L. et al. Maternal age and risk of stillbirth: a systematic review. Canadian Medical Association Journal, Otawa, v. 178, n. 2, p. 165-72, Jan. 2008.

JACOBSSON, B.; LADFORS, L.; MILSOM, I. Advanced maternal age and adverse perinatal outcome. Obstetrics \& Gynecology, New York, v. 104, n. 4, p. 727-733, Oct. 2004.

JOBIM, R.; AERTS, D. Mortalidade infantil evitável e fatores associados em Porto Alegre, Rio Grande do Sul, Brasil, 20002003. Cadernos de Saúde Pública, Rio de Janeiro, v. 24, n. 1, p. 179-187, jan. 2008.

JOLLY, M. C. et al. Obstetric risks of pregnancy in women less than 18 years old. Obstetrics and Gynecology, United States, v. 96, n. 6, p.962-966, Dec. 2000.

KING, J.C. The risk of maternal nutritional depletion and poor outcomes increases in early or closely spaced pregnancies. Journal of Nutrition, Philadelphia, v. 133, n. 5, p. 1.732S-1.736S, May 2003. Suplemento 2.

KRISTENSEN, S. et al. Impact of advanced maternal age on neonatal survival of twin small-for-gestational-age subtypes. Journal of Obstetrics and Gynaecology Research, Tokyo, v. 33, n. 3, p. 259-265, Jun. 2007.

LAWLOR, D. A.; SHAW, M. Too much too young? Teenage pregnancy is not a public health problem. International Journal of Epidemiology, Oxford, v. 31, n. 3, p. 552554, Jun. 2002.

LEITE, I. da C.; RODRIGUES, R. N.; FONSECA, M. C. Fatores associados com o comportamento sexual e reprodutivo entre adolescentes das regiões Sudeste e Nordeste do Brasil. Cadernos de Saúde Pública, Rio de Janeiro, v. 20, n. 2, p. 474 - 481, mar./ abr. 2004.

LERIDON, H. Can assisted reproduction technology compensate for the natural decline in fertility with age? A model assessment. Human Reproduction, Oxford, v. 19, n. 7, p.1548-1553, Jul. 2004.
LUKE, B.; BROWN, M. B. Elevated risks of pregnancy complications and adverse outcomes with increasing maternal age. Human reproduction, Oxford, v. 22, n. 5, p. 1264-1272, May 2007.

LUTHER, J. et al. Maternal and fetal growth, body composition, endocrinology, and metabolic status in undernourished adolescent sheep. Biology of Reproduction, Champaign, v. 77, n. 2, p. 343-350, Aug. 2007.

MACHADO, C. J.; HILL, K. Determinants of neonatal and post-neonatal mortality in the City of São Paulo. Revista Brasileira de Epidemiologia, São Paulo, v. 6, n. 4, p. 345-357, Dec. 345-58. 2003.

MAHESHWARI, A.; HAMILTON, M.; BHATTACHARYA, S. Effecty of female age on the diagnostic categories of infertility. Human Reproduction, Oxford, v. 23, n. 3, p.538-542, Mar. 2008.

MARASINGHE, J. P.; KARUNANANDA, S. A.; AMARASINGHE, A. A. The mature gravida: a different approach. European Journal of Obstetrics \& Gynecology and Reproductive Biology, Amsterdan, v. 135, n. 1, p. 136, Nov. 2007.

MARCONDES, G. S. Recasamento formal no Brasil: alguns achados do registro civil. Textos Nepo, Campinas, v. 58, p. 1-39, set. 2008.

MINAGAWA, A. T. et al. Baixo peso ao nascer e condições maternas no pré-natal. Revista da Escola de Enfermagem, São Paulo, v. 40, n. 4, p. 548-554, dez. 2006.

MOHSIN, M.; BAUMAN, A. E.; JALALUDIN, $B$. The influence of antenatal and maternal factors on stillbirths and neonatal deaths in New South Wales, Australia. Journal of Biosocial Science, Oxford, v. 38, n. 5, p. 643-657, Sep. 2006.

MONTAN, S. Increased risk in the elderly parturient. Current Opinion in Obstetrics \& Gynecology, London, v. 19, n. 2, p. 110112, Apr. 2007.

NABUKERA, S. K. Interpregnancy interval and subsequent perinatal outcomes among women delaying initiation of childbearing. 
Journal of Obstetrics and Gynaecology Research, Tokyo, v. 34, n. 6, p. 941-947, Dec. 2008.

O'LEARY, C.M. et al. Changing risks of stillbirth and neonatal mortality associated with maternal age in Western Australia 1984-2003. Paediatric and Perinatal Epidemiology, England, v. 21, n. 6, p. 541549, Nov. 2007.

OLESZCZUK, J. J.; KEITH, L. G.; OLESZCZUK, A. K. The paradox of old maternal age in multiple pregnancies. Obstetrics and Gynecology Clinics of North America, Philadelphia, v. 32, n. 1, p. 69-80, Mar. 2005.

OLIVEIRA, J. de C. Perfil socioeconômico da maternidade nos extremos do período reprodutivo. Rio de Janeiro: IBGE, 2005.

OLIVEIRA, M. C. F. A.; MARCONDES, G. dos S. Contabilizando perdas e ganhos: maternidade, trabalho e conjugalidade no pós-feminismo. In: ENCONTRO NACIONAL DE ESTUDOS POPULACIONAIS, 14. Anais... Caxambu: Abep, 2004.

PANTELIDES, E. A. Aspectos sociales del embarazo y la fecundidad adolescente en América Latina. Notas de Población, Santiago de Chile, v. 31, n. 78, p. 7-34. Dec. 2004.

PASQUAL-OTTO, F. F.; BORGES JUNIOR, E.; PASQUALOTTO, E. B. The male biological clock is ticking: a review of the literature. São Paulo Medical Journal, São Paulo, v. 126, n. 3, p. 197-201, May 2008.

PHIPPS, M. G.; SOWERS, M. Defining early adolescent childbearing american. Journal of Public Health, n. 92, p. 125-128. 2002.

RAATIKAINEN, K.; HEISKANEN, N.; HEINONEN, S. Induced abortion: not an independent risk factor for pregnancy outcome, but a challenge for health counseling. Annals of Epidemiology, New York, v. 16, n. 8, p. 587-592, Aug. 2006.

RAYAMAJHI, R.; THAPA, M.; PANDE, S. The challenge of grandmultiparity in obstetric practice. Kathmandu University Medical Journal, Nepal, v. 4, n. 1, p. 70-74, Jan./ Mar. 2006.
REDE INTERAGENCIAL DE INFORMAÇÕES PARA A SAÚDE. IDB-2007. Indicadores de mortalidade. Brasília: Rede Interagencial de Informações para a saúde. 2008. Disponível em: <http://www.datasus.gov.br/idb> . Acesso em: 02 fev. 2009.

REIME, B.; SCHÜCKING, B. A.; WENZLAFF, $P$. Reproductive outcomes in adolescents who had a previous birth or an induced abortion compared to adolescent's first pregnancies. BMC Pregnancy Childbirth, England, v.8, n.4, Jan. 2008.

ROTHER, E. T. Revisão sistemática X revisão narrativa. Acta Paulista de Enfermagem, São Paulo, v. 20, n. 2, June 2007.

SCHOLL, T. O. et al. Maternal growth during pregnancy and the competition for nutrients. American Journal of Clinical Nutrition, New York, v. 60, n. 2, p. 183-8, Aug. 1994.

SCHOLL, T. O.; HEDIGER, M. L.; ANCES, I. G. Maternal growth during pregnancy and decreased infant birth weight. American Journal of Clinical Nutrition, New York, v. 51, n. 5, p.790-793, May 1990.

SENESI, L. G. et al. Morbidade e mortalidade neonatais relacionadas à idade materna igual ou superior a 35 anos, segundo a paridade. Revista Brasileira de Ginecologia e Obstetrícia, Rio de Janeiro, v. 26, n. 6, p. 477-482, jul. 2004.

SHARMA, V. et al. Young maternal age and the risk of neonatal mortality in rural Nepal. Archives of Pediatrics \& Adolescent Medicine, Chicago, v. 162, n. 9, p. 828-835, Sep. 2008.

SILVA, A. A. et al. Young maternal age and preterm birth. Paediatric and Perinatal Epidemiology, England, v. 17, n. 4, p. 332339, Oct. 2003.

SIMÃO, A. B. A primeira relação sexual, o primeiro casamento e o nascimento do primeiro filho: um estudo quantitativo e qualitativo de duas coortes de mulheres em Belo Horizonte. Tese (Doutorado em Demografia) - Centro de Desenvolvimento e Planejamento Regional, Universidade Federal de Minas Gerais, Belo Horizonte, 2005. 
SIMÕES, V. M. F. et al. Características da gravidez na adolescência em São Luís, Maranhão. Revista de Saúde Pública, São Paulo, v. 37, n. 5, p. 559-565, out. 2003.

SINGH, S.; DARROCH, J. E. Adolescent pregnancy and childbearing: levels and trends in developed countries. Family Planning Perspectives, New York, v. 32, n.1, p.14-23, Jan./Feb. 2000.

STEIN, Z.; SUSSER, M. The risks of having children in later life. Social advantage may make up for biological disadvantage. BMJ, London, v. 320, n. 7.251, p. 1.681-1.682, Jun. 2000.

TAIN, L. Um filho quando eu quiser? O caso da França contemporânea. Estudos Feministas, Florianópolis, v. 13, n. 1, p. 5367, jan./ abr. 2005.

TOUGH, S. C. et al. Delayed childbearing and its impact on population rate changes in lower birth weight, multiple birth, and preterm delivery. Pediatrics, Illinois, v. 109, n. 3, p. 399-403, Mar. 2002.
WORLD HEALTH ORGANIZATION. Adolescent pregnancy: issues in adolescent health and development. Geneva, Switzerland: World Health Organization, 2004. Disponível em: <http://whqlibdoc. who.int/publications/2004/9241591455_eng. pdf > . Acesso em: 15 nov. 2008.

Assisted reproduction in developing countries: facing up to the issues. Progress in Reproductive Health Research, Wolrd Health Organization, 2003. Disponível em: <http://www.who.int/ reproductive-health/hrp/progress/63/63. pdf>. Acesso em: 28 jan. 2009.

YÁNEZ, L. E. O. Primigesta de edad avanzada. Revista de Obstetricia y Ginecología de Venezuela, Caracas, v. 67, n. 3, p.152-166, Sep. 2007.

YAZAKI, L. M. Análise da fecundidade no Estado de São Paulo. São Paulo em Perspectiva, São Paulo, v. 22, n. 1, p. 48-65, jan./jun. 2008.

\section{Resumen}

Edad materna y mortalidad infantil: ¿efectos nulos, biológicos o socioeconómicos?

Hay controversias en cuanto a si los extremos de la edad reproductiva femenina agregan el mayor conjunto de factores asociados a la mortalidad infantil, y si para los hijos de madres de menos de 20 años y desde los 35 años de edad hay evidencias de mayores probabilidades de ocurrencia de muerte infantil. Para las madres adolescentes, hay un importante debate en cuanto a si los resultados obstétricos adversos alcanzados se deben a la inmadurez biológica, a las condiciones socioeconómicas desfavorables, o si hay nulidad del efecto de la corta edad sobre las probabilidades de ocurrencia de mortalidad infantil. Para madres de edades avanzadas, se discute si el aumento en la edad materna, a pesar de los avances en el campo de la medicina, está asociado al crecimiento de la vulnerabilidad a la mortalidad infantil, si las mejores condiciones socioeconómicas atenúan el efecto de las comorbidades maternas comunes a la edad de 35 años o más, o incluso si la maternidad tardía no ejerce algún efecto sobre la ocurrencia de mortalidad infantil. Sobre la base de evidencias contrastantes acerca de las probabilidades de ocurrencia de mortalidad infantil debida a la edad materna, este trabajo tiene como objetivo presentar y discutir los principales argumentos evocados por la literatura. En el caso de la maternidad precoz y la tardía, hay evidencias que corroboran ambas hipótesis de la plausibilidad biológica y socioeconómica, y de la nulidad del efecto de la edad materna. La variedad de metodologías empleadas para el estudio de la asociación entre edad de la madre 
al tener el hijo y la mortalidad infantil, además de la falta de uniformidad en la definición de los grupos etarios entre los diversos estudios, dificulta una identificación más precisa acerca de esta vulnerabilidad.

Palabras-clave: Mortalidad infantil. Edad materna. Efectos biológicos. Efectos socioeconómicos. Tendencias de fecundidad.

\section{Abstract \\ Mothers' ages and infant mortality rates: null, biological or socioeconomic effects?}

There are controversies as to whether extreme ages of women upon giving birth bring with them a greater number of factors associated with infant mortality rates and whether there is evidence of higher mortality rates among children of new mothers below age 20 or over age 34. Regarding teenage mothers, a heated debate is in course as to whether adverse obstetric results that occur are due to biological immaturity, to unfavorable socioeconomic situations, or if there is null effect of a woman's young age on the chances of infant mortality. One question involving older mothers at childbirth is whether, despite the inroads made in medicine, this age can be associated with higher vulnerability to infant mortality. Another factor is whether more favorable socioeconomic conditions reduce the effect of the most common infirmities seen in mothers who gave birth at age 35 or over. There is also the question as to whether late affects the occurrence of infant mortality. On the basis of contrasting evidence regarding the levels of child mortality as correlated with mothers' age, this article presents and discusses the main arguments found in the literature. In the case of both early and late childbirth, there are indications that back up not only the hypotheses of the biological and socioeconomic plausibility of infant mortality, but also the position that there is no effect of mothers' ages on this factor. Two factors hamper more precise identification regarding this vulnerability. First there is the variety of methods used to study the relationship between mother's age when giving birth, on the one hand, and infant mortality, on the other. Secondly, there is lack of uniformity among the studies in defining age groups.

Keywords: Infant mortality. Mothers' age. Biological effects. Socioeconomic effects. Fertility trends. 